\title{
Geographische Informationssysteme (GIS) des Österreichischen Statistischen Zentralamtes (ÖSTAT) und der Europäischen Union
}

\author{
Helmut Desoye \\ Österreichisches Statistisches Zentralamt, Wien
}

Zusammenfassung: Im ÖSTAT wird das Geographische Informationssystem STATGIS entwickelt, in der EU besteht seit 1992 das GIS der Europäischen Kommission (GISCO). STATGIS wird es in den Richtmaßstäben $1: 500000,1: 5000$ und $1: 50000$ geben. Kernstück ist das STATGIS 1 : 5 000, das auf Adreß- bzw. Gebäudekoordinaten basiert und statistische Auswertungen für beliebige räumliche Gebilde ermöglicht, vorbehaltlich der Datenschutz bleibt gewahrt. Für die anderen Richtmaßstäbe sollen u.a. Standardprodukte angeboten werden. GISCO enthält eine große Menge topographischer und thematischer Informationen und wird ständig verbessert. Gesamtziel ist die Schaffung eines Europäischen Datenbanksystems für Geoinformation in mehreren Stufen, wobei auch ein Verbund mit nationalen und regionalen Informationssystemen hergestellt werden soll.

\begin{abstract}
Within the Austrian Central Statistical Office a Geographical Information System (GIS) with 3 basic scales (1:500 000, $1: 5$ 000, $1: 50$ 000) is in the state of development. The scale 1:5000 is based on coordinates of buildings and will allow the publication of statistical data for optional territories. GISCO is the GIS of the European Commission. It exists since 1992 and is continuously improved. It is intended to create a European Data Bank System for Geographical Information in several steps whereby a connection to national and regional information systems shall be installed.
\end{abstract}

Schlüsselwörter: STATGIS, Geocodierung von Adressen und Gebäuden, Gebäuderegister, Layer, UTM-Netz, GISCO, Europäisches Datenbanksystem, REGIO.

\section{Einleitung}

Im ersten Hauptkapitel wird die Entwicklung des „STATGIS“ des ÖSTAT, im zweiten das „GISCO“ genannte Geographische Informationssystem der Europäischen Kommission und die beabsichtigte Entwicklung eines europäischen GIS-Netzwerkes behandelt.

\section{Die Entwicklung des Geographischen Informationssys- tems STATGIS des ÖSTAT}

Mit Hilfe der weltweit verwendeten Softwareprodukte Arc/Info und Arc/View wird seit rund zwei Jahren im ÖSTAT ein Geographisches Informationssystem, das als „STATGIS“ bezeichnet wird, in drei Richtmaßstäben entwickelt. Diese Richtmaßstäbe sind: 


$$
\begin{aligned}
& 1: 500000=\text { STATGIS } / 500 \\
& 1: 5000=\text { STATGIS } / 5 \\
& 1: 50000=\text { STATGIS } / 50
\end{aligned}
$$

Im folgenden werden STATGIS/500 und STATGIS/50 nur kurz behandelt, STATGIS/5 wird hingegen ausführlicher beschrieben.

\subsection{STATGIS/500}

Diese Version des GIS, dessen Einrichtung bis zum 1. Quartal 2000 vorgesehen ist, wird Auswertungen ermöglichen, die für das ganze Bundesgebiet über die Länder bis hinab zu Einheiten von der Größe der NUTS 3 reichen. Die Datenbasis wird in der Regel die Gemeindeebene (= NUTS 5) sein, allenfalls auch noch kleinere Einheiten wie die statistischen Zählsprengel. Da die Daten für Gemeinden und Zählsprengel jedoch für jedermann zugänglich sind, können GIS-Auswertungen auch von Institutionen oder Firmen außerhalb des ÖSTAT durchgeführt werden. Es ist daher beabsichtigt, für diesen Grundmaßstab seitens des Amtes einige Standardprodukte anzubieten, die grundlegende Auswertungen über Österreich betreffen. Im Rahmen des STATGIS/500 wurden bislang Gitternetzlayer mit Maschen von 10, 5 und 2,5 km Seitenlänge installiert, welchen die Gemeinden oder Zählsprengel aufgrund ihrer Siedlungsschwerpunkte zugeordnet wurden (siehe Abb. 1 und 2).

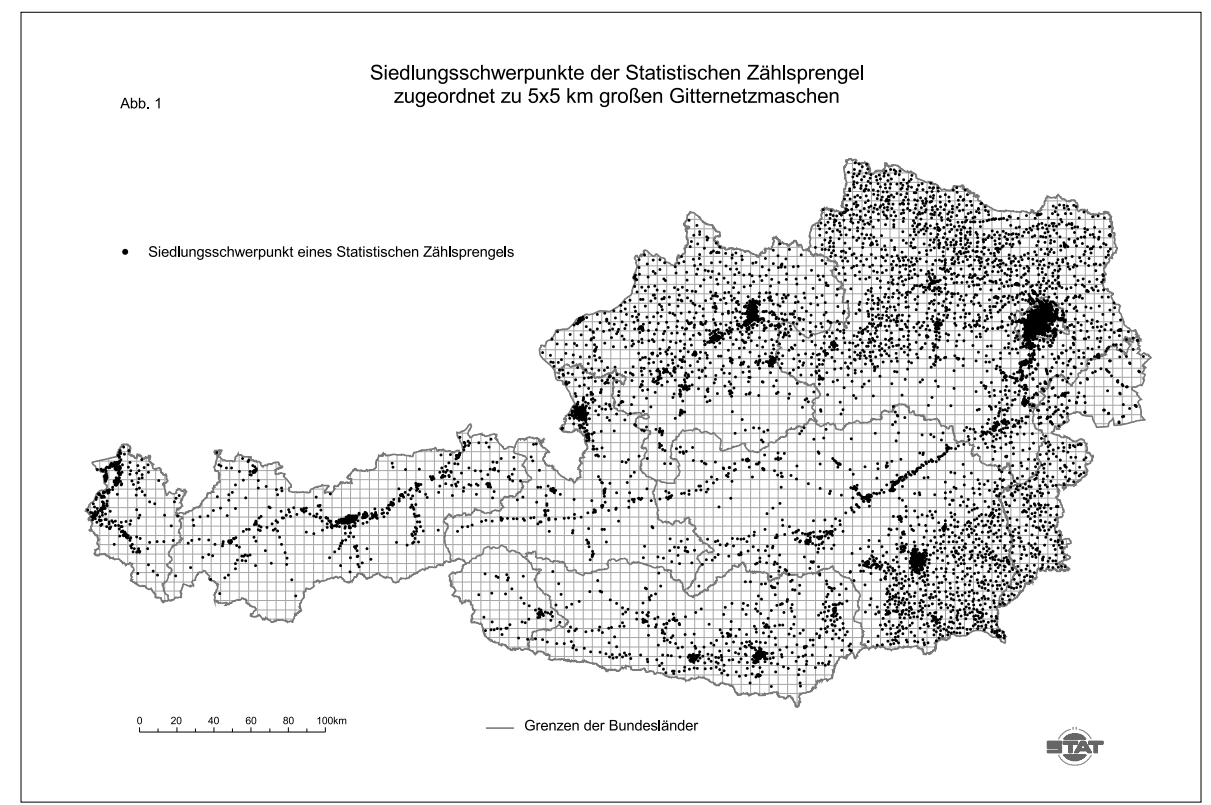

\subsection{STATGIS/5}

Das GIS in diesem Richtmaßstab ist als Kernstück des STATGIS anzusehen. Es soll nämlich auf einfache Weise Auswertungen über beliebig abgrenzbare kleinräumige territoriale Einheiten zulassen. Die Datenbasis sind dabei alle Daten, die mit Adressen oder 


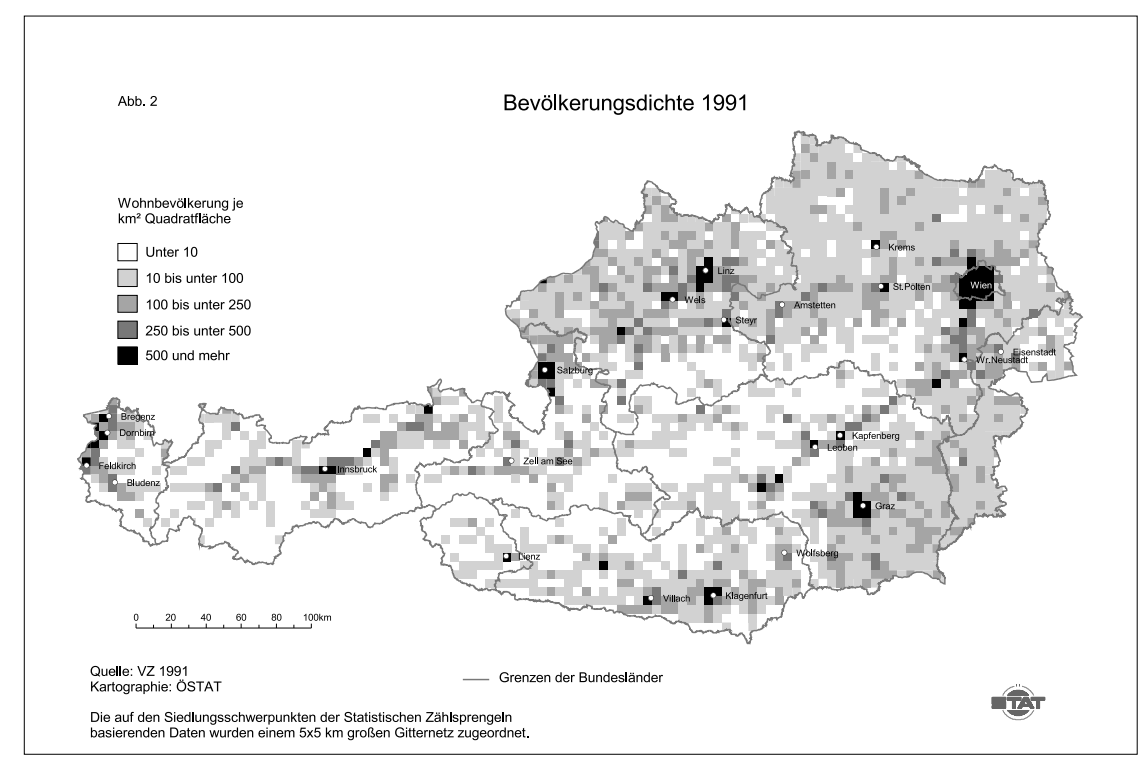

Gebäuden verknüpft sind. Voraussetzung ist die Geocodierung dieser Adressen bzw. Gebäude, die aufgrund eines Abgleiches des Gebäuderegisters des ÖSTAT mit den Adreßdateien des Bundesamtes für Eich- und Vermessungswesen erfolgen wird. Für einen Großteil Österreichs werden Auswertungen bereits aus der Großzählung 2001 möglich sein, für den Rest spätestens 2005.

Wenn ein Auftraggeber die Umgrenzungen der von ihm gewünschten Gebiete (z.B. Baublöcke) mittels Koordinaten definiert, werden alle Adressen bzw. Gebäude, die innerhalb dieser Gebiete liegen, diesen automatisch zugeordnet. Es ist auch möglich, Gebiete aufgrund vorgegebener Merkmale abzugrenzen.

Es ist naheliegend, daß ein derartiges GIS von Privaten nicht geführt werden kann, weil das ÖSTAT Daten je Koordinate (Adresse oder Gebäude) aus Geheimhaltungs- bzw. Datenschutzgründen nicht weitergeben darf. Was den Aufwand für das Amt allerdings sehr verringern könnte, wäre die entgeltliche Überlassung des (jeweils benötigten Teiles) des Gebäuderegisters samt Koordinaten an den Auftraggeber, der damit seine Gebiete samt Plausläufen selbst definieren kann und vom ÖSTAT lediglich die Daten dazugespielt bekommt. Um zu verhindern, daß die vom Auftraggeber definierten Gebiete jene Grenzen unterschreiten, die - merkmalsabhängig - für die statistische Geheimhaltung festgelegt sind, würde das ÖSTAT dem Auftraggeber gewisse Grundzahlen bekanntgeben, also z.B. die Wohnbevölkerung für Baublöcke. Da unter 30 Einwohnern keine Strukturdaten mehr bekanntgegeben werden dürfen, könnte der Auftraggeber dann seine Baublöcke entsprechend aggregieren, um Daten zu erhalten (siehe Abb. 3).

Für das GIS/5 sollen Routinen ausgearbeitet werden. Dazu gehört die Definition von Standardabfragepaketen für Daten bzw. Datenkombinationen, zunächst aufgrund der Bedarfsabschätzung und später auch aufgrund von Erfahrung mit Aufträgen. Ein sehr wichtiger Gesichtspunkt für ein GIS in diesem großen Maßstab ist auch die genaue Untersuchung, wie die Datengeheimhaltung gewahrt werden kann.

Hinsichtlich des STATGIS/5 wurden bereits ziemlich eingehende Erfahrungen durch 


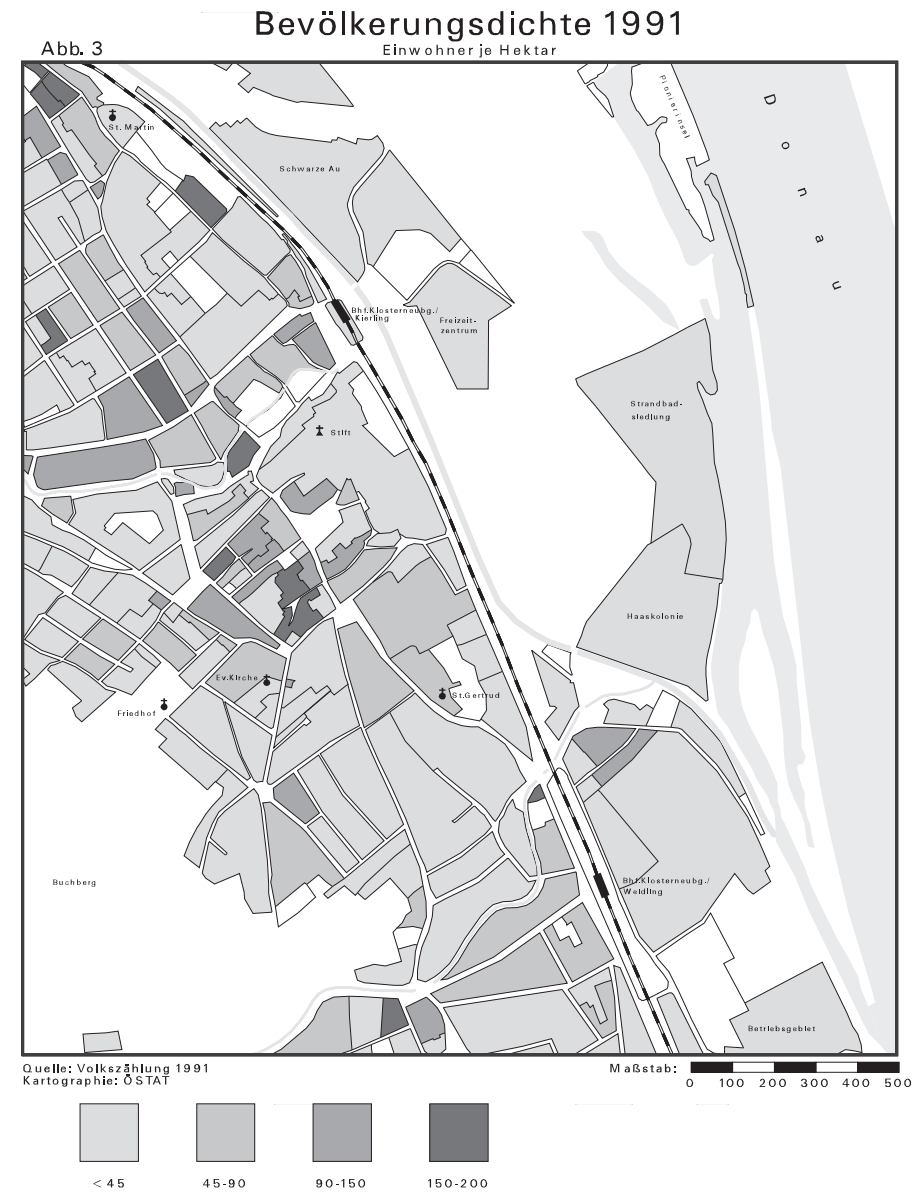

Probeauswertungen gewonnen. Die methodischen Vorarbeiten werden bis zur ersten Auswertungsphase der Großzählung 2001 abgeschlossen sein, also bis zur Jahreswende 2001/2002.

\subsection{STATGIS/50}

GIS-Auswertungen im Maßstab 1 : 50000 werden auf der Basis der statistischen Zählsprengel bereits jetzt von mehreren Firmen durchgeführt. Auswertungen durch das Amt wären aber dann gerechtfertigt, wenn als Datenbasis nicht die Zählsprengel, sondern wieder die geocodierten Adressen oder Gebäude herangezogen werden. Diese könnten den Maschen des UTM-Netzes als standardisierte Auswertungseinheiten zugeordnet werden. Auch hier ist es wiederum denkbar, daß nur die Daten für die UTM-Maschen vom ÖSTAT geliefert werden, wobei Vorkehrungen für die Geheimhaltung bei zu kleinen Grundgesamtheiten zu treffen sind. Es wird zu prüfen sein, ob Standardauswertungen auch im GIS/50 angeboten werden können. Die methodischen Vorarbeiten könnten ebenfalls bis zur Jahreswende 2001/2002 abgeschlossen sein. 


\section{Das Geographische Informationssystem der Europäi- schen Kommission (GISCO) und die beabsichtigte Ent- wicklung eines europäischen GIS-Netzwerkes}

Die räumliche Dimension des Europäischen Statistischen Systems gewinnt als Entscheidungshilfe in einer Vielzahl der Zuständigkeitsbereiche der Kommission zunehmend an Bedeutung. Im Rahmen des 1992 ins Leben gerufenen Projekts GISCO hat Eurostat eine Referenzdatenbank aufgebaut, in der eine große Menge topographischer und thematischer Geoinformationen für die gesamte Kommission gespeichert ist. Diese Aufgabe konnte nur in Zusammenhang zwischen GISCO und dem Netzwerk seiner Nutzer in den Generaldirektionen der Kommission, die die GIS-Technologie anwenden, bewältigt werden.

Naturgemäß ist man bestrebt, GISCO ständig zu verbessern, wozu es diverse Studien gibt. Man kann drei Arten von Daten unterscheiden, nämlich geographische Referenzdaten (Verwaltungsgrenzen, Gewässer, Bodenerhebungen, Verkehrsnetze usw.), thematische oder gemeinsame Daten, das sind Geodaten und Attributdaten, die von mehreren Generaldirektionen gemeinsam genutzt werden und besondere sektorspezifische Daten, das sind Geodaten und Attributdaten, die von einer einzelnen Generaldirektion genutzt werden (z.B. Daten über den Zustand der Wälder). Erstere beiden Datenarten sollen aufgrund einer Studie bei Eurostat verwaltet werden.

Gesamtziel ist die Schaffung eines Europäischen Datenbanksystems für Geoinformation in mehreren Stufen, wobei auch ein Verbund mit nationalen und regionalen Informationssystemen hergestellt werden soll.

GISCO gliedert seine Daten in einer thematisch-hierarchischen Struktur, wobei man zwei hierarchische Ebenen, nämlich die der Themen (z. B. Infrastruktur), die ihrerseits wieder Themenbereichen (z.B. Topographische Daten) angehören, und jene der Schichten unterscheidet (z.B. Flughäfen, Häfen).

Aus der Datenbank GISCO stehen für die Allgemeinheit folgende Daten auf CDROM im Arc/Info-Exportformat, gegen zusätzliche Gebühr auch auf anderen Datenträgern, zur Verfügung (Details wären dem Eurostat-Katalog zu entnehmen):

\subsection{Themenbereich Verwaltungsgrenzen}

Enthält die Themen: Verwaltungsgrenzen (mit den Schichten NUTS-Regionen der EU und Verwaltungsregionen für Gesamteuropa)

Welt-Datenbanken (Schicht: Welt-Verwaltungsregionen)

\subsection{Themenbereich Topographische Daten}

Enthält die Themen: Verwaltungsgrenzen (wie unter 2.1 beschrieben)

Infrastruktur (Schichten: Flughäfen, Häfen, Siedlungen)

Hydrographie (Binnengewässer, Wasserscheiden)

Höhenmessung (Schicht: Digitales Höhenmodell-Gesamteur.)

Welt-Datenbanken (Schicht: Höhenmessung-Welt) 


\subsection{Themenbereich Landressourcen, Natürliche Ressourcen, Umwelt}

Enthält die Themen: Verwaltungsgrenzen (wie unter 2.1 beschrieben) Landressourcen (Schichten: Klima, Fischgründe)

Natürliche Ressourcen (Biogeographische Zonen, Landschaften-Gesamteuropa, Natürliche potentielle Vegetation)

Umwelt (Küstenerosion, Bodengüte, Bodenerosionsrisiko)

Welt-Datenbanken (Schicht:Fischgründe, Welt).

Durch die Verbindung der Datenbank GISCO mit der Datenbank REGIO, deren Daten auf unterschiedlichen Datenträgern erhältlich sind, können sozio-ökonomische Daten aus folgenden Bereichen von REGIO für die Ebenen NUTS 1 bis NUTS 3 kartographisch dargestellt werden:

Bevölkerung, - Volkswirtschaftliche Gesamtrechnung, - Arbeitslosigkeit, - Erwerbstätigkeit, - Energiestatistiken, - Landwirtschaft, - Verkehr, - Forschung und Entwicklung.

Da die Nutzung von GIS zur Analyse und Darstellung der Daten innerhalb der nationalen statistischen Systeme und des gemeinschaftlichen Systems ständig weitere Verbreitung findet, liegt es nun an der Gemeinschaft, sich zu organisieren, wobei Eurostat die Initiative für ein solches Projekt ergreifen muß. Ziel eines solchen Projektes wäre die Einrichtung eines europäischen GIS-Netzwerkes für die Statistik, das die gemeinsame Nutzung und den Austausch von Informationen und Verfahren unter seinen Mitgliedern ermöglicht. Vor Inangriffnahme eines solchen Projektes müssen jedoch die Zielsetzungen und die Durchführbarkeit in einer Studie analysiert werden. Diese muß folgende, auf den Nutzerbedarf ausgerichtete Phasen berücksichtigen:

- Reiner Austausch von Informationen, Daten, Metadaten, Verfahren usw.;

- Definition, Austausch und Aktualisierung eines Grunddatenbestandes für die gesamte EU;

- Verteilte gemeinsame Referenzdatenbank für die Netzmitglieder unter der Voraussetzung, daß über Basisdaten, eine gemeinsame Datenbankstruktur, Metadatenstandards, die Aufteilung der Zuständigkeiten usw. Einigung erzielt wird.

Adresse des Autors:

Helmut Desoye

Österreichisches Statistisches Zentralamt

Hintere Zollamtstraße 2B

A-1033 Wien

Tel. $+43171128 / 7393$

Elec. Mail: helmut.desoye@oestat.gv.at 